\title{
A Discussion on Formulas of Seismic Hydrodynamic Pressure
}

\author{
Liu Haiming ${ }^{1, a}$, Tao Xiaxin ${ }^{2, b}$ \\ ${ }^{1}$ China Merchants Chongqing Communication Research \& Design Institute Co., Ltd, State Key \\ Laboratory of Bridge Engineering Structural Dynamics, Chongqing, 400067, China \\ ${ }^{2}$ School of Civil Engineering, Harbin Institute of Technology, Harbin, 150090, China \\ aliuhaiming@cmhk.com, btaoxiaxin@aliyun.com
}

Keywords: Hydrodynamic pressure, Seismic design, Highway bridge, Guidelines

Abstract. The origin and simplification of formulas for seismic hydrodynamic pressure in the existing guidelines for seismic design of highway bridges of China is reviewed. Five differences from Japanese specifications that is Chinese guidelines come from, are pointed out. A suggestion to modify the formulas is finally presented.

\section{Introduction}

Recently, some researchers mentioned the fact that values of seismic hydrodynamic pressure from the existing guidelines for seismic design of highway bridges of China [1] are much lower than those from Japanese design specifications for highway bridges, part V seismic design [2]. The authors of this paper, review the codes, search the origin of the formulas, point out the cause of the difference and then present a modification suggestion.

\section{Origin of the formulas in seismic design code of highway engineering of China}

The seismic hydrodynamic pressure was firstly taken into account for the situation with water depth larger than 5 meters in seismic design code of highway bridges of China in 1989 [3]. The formulas for three value intervals of pier size to water depth ratio are as following.

$$
\begin{aligned}
& \left.\mathrm{E}_{\mathrm{w}}=0.151-\frac{\mathrm{b}}{4 \mathrm{~h}}\right) \mathrm{C}_{\mathrm{i}} \mathrm{K}_{\mathrm{h}} \xi_{\mathrm{h}} \gamma_{\mathrm{w}} \mathrm{b}^{2} \mathrm{~h}, \quad \text { if } \quad \frac{b}{h} \leq 2.0 \\
& E_{w}=0.075 C_{i} K_{h} \xi_{h} \gamma_{w} b^{2} h, \quad \text { if } \quad 2.0<\frac{b}{h} \leq 3.1 \\
& E_{w}=0.24 C_{i} K_{h} \gamma_{w} b h^{2}, \quad \text { if } \quad \frac{b}{h}>3.1
\end{aligned}
$$

where $E_{\mathrm{w}}$ is the resultant force of total seismic hydrodynamic pressure acted on the pier at height of $h / 2$ $(\mathrm{kN}), C_{i}$ is importance factor, $K_{\mathrm{h}}$ is the design lateral seismic coefficient defined as the ratio of design horizontal basic acceleration to gravity acceleration, $h$ is the water depth (m), $b$ is the pier width in the direction perpendicular to the direction of hydrodynamic pressure $(\mathrm{m}), \xi_{\mathrm{h}}$ is section shape factor with value 1.0 for rectangular and 0.8 for circular, $\gamma_{w}$ is unit weight of water $\left(\mathrm{kN} / \mathrm{m}^{3}\right)$.

One can know from the commentary of the specifications that the origin of these formulas comes from Japanese 1980 design specifications for highway bridges, Part V seismic design [4]. The formulas in Japanese code are as follows [5].

$$
P=\frac{3}{4} k_{h} w_{0} A_{0} h \frac{b}{a}\left(1-\frac{b}{4 h}\right), \quad \text { if } \quad \frac{b}{h} \leq 2.0
$$




$$
\begin{aligned}
& P=\frac{3}{4} k_{h} w_{0} A_{0} h \frac{b}{a}\left(0.7-\frac{b}{10 h}\right), \quad \text { if } \quad 2.0<\frac{b}{h} \leq 4.0 \\
& P=\frac{9}{40} k_{h} w_{0} A_{0} h \frac{b}{a}, \quad \text { if } \quad 4.0<\frac{b}{h}
\end{aligned}
$$

where $P$ is the same as $E_{\mathrm{w}}$ in Eq. 1 but at height of $3 h / 7, k_{\mathrm{h}}$ is the product of $K_{\mathrm{h}}$ in Eq. 1 with a comprehensive factor $C_{\mathrm{s}}$ and an important factor as $C_{\mathrm{i}}$ in Eq. 1, in addition of regional and site factor, $w_{0}$ is the same as $\gamma_{\mathrm{w}}$ in Eq.1, $A_{0}$ is area of the pier section at the height of $3 h / 7, h$ and $b$ are the same as in Eq. 1, $a$ is the length of other side of rectangular section of the pier.

\section{Origin of the formulas in Japanese design specifications for highway bridges}

According the commentary of Japanese design specifications for highway bridges, Part V seismic design $[2,4,5]$, the above formulas come from the suggestion by the Goto and Toki [6]. From the following integrating equation on velocity potential in cylindrical coordinate system, Eq.7 and the boundary conditions in Eq.8, Eq. 9, Eq.10 and Eq.11, dynamic pressure on a rigid cylinder surface in three dimensional analysis can be evaluated by Eq. 12 .

$$
\begin{aligned}
& \frac{\partial^{2} \phi}{\partial r^{2}}+\frac{1}{r} \frac{\partial \phi}{\partial r}+\frac{1}{r^{2}} \frac{\partial^{2} \phi}{\partial \theta^{2}}+\frac{\partial^{2} \phi}{\partial z^{2}}-\frac{1}{c^{2}} \frac{\partial^{2} \phi}{\partial t^{2}}=0 \\
& \left(\frac{\partial \phi}{\partial r}\right)_{r=a}=-\frac{\partial y}{\partial t} \cos \theta \\
& \left(\frac{\partial \phi}{r \partial \theta}\right)_{\theta=0}=\left(\frac{\partial \phi}{r \partial \theta}\right)_{\theta=\pi}=0 \\
& \left(\frac{\partial \phi}{\partial z}\right)_{z=0}=0 \\
& \left(g \frac{\partial \phi}{\partial z}+\frac{\partial^{2} \phi}{\partial t^{2}}\right)_{z=h}=0 \\
& P_{y}=k_{0} \gamma_{w} \pi a^{2} \sum_{m=1}^{\infty} \frac{(-1)^{m-1}}{\alpha_{m} h} \frac{4}{\alpha_{m} a} \frac{K_{1}\left(\alpha_{m} a\right)}{K_{0}\left(\alpha_{m} a\right)+K_{z}\left(\alpha_{m} a\right)} \cos \alpha_{m} z
\end{aligned}
$$

where $k_{0}$ is the lateral seismic coefficient, $\gamma_{\mathrm{w}}$ and $h$ are the same as in Eq.1, $a$ is the radius of the cylinder, others are coefficients and functions not illustrated here.

In simplification, a cubic expression is taken for distribution of the hydrodynamic pressure with the ordinate $y$, in the case of the cylinder moving in finite mass of water, the following formula is then derived. 


$$
P_{y}=k_{o} \gamma_{w} \pi a^{2}\left(1-\frac{a}{2 h}\right) \sqrt[3]{1-\frac{z}{h}}
$$

Integral of the term containing $z$ from 0 to $h$ can be obtained as

$$
\int_{0}^{h} \sqrt[3]{1-\frac{z}{h}} d z=\frac{3}{4} h
$$

The formula of hydrodynamic pressure is then finally derived as follows.

$$
P=\frac{3}{4} k_{0} \gamma_{w} \pi a^{2} h\left(1-\frac{a}{2 h}\right)
$$

One can see that it is exactly the same as Eq. 4, if $a$ here equals $b / 2$ in Eq. $4, \pi a^{2}$ equals $A_{0}=a b$ in Eq. 4.

\section{Formulas in the existing guidelines for seismic design of highway bridges and some thoughts for a modification}

The formulas on hydrodynamic pressure stipulated in the existing guidelines for seismic design of highway bridges of China [1], are the following three.

$$
\begin{array}{ll}
E_{w}=0.15\left(1-\frac{b}{4 h}\right) C_{i} A \xi_{h} \gamma_{w} b^{2} h / g, & \text { if } \quad \frac{b}{h} \leq 2.0 \\
E_{w}=0.075 C_{i} A \xi_{h} \gamma_{w} b^{2} h / g, & \text { if } \quad 2.0<\frac{b}{h} \leq 3.1 \\
E_{w}=0.24 C_{i} A \gamma_{w} b h^{2} / g, & \text { if } \quad \frac{b}{h}>3.1
\end{array}
$$

where all parameters here are the same as in Eq. 1, Eq. 2 and Eq. 3 respectively, except for the $A$ is horizontal design basic peak ground acceleration, and there must be a $/ g$, the gravity acceleration, added from the definition of seismic coefficient in Eq. 1.

Pay attention on the fact that $A_{0}=a b$ for pier with rectangular section, one can see from the above formulas that the formulas in Chinese guidelines are almost the same as in Japanese specifications, with five differences. First, the value of the first consistent at the right side in Eq. 4 is five times as one in Eq. 1, since a comprehensive factor $C_{\mathrm{Z}}=0.2$ is taken for both seismic load and earth pressure in Chinese code. Second, the second cutoff value of the three ratio intervals is 4.0 in Eq. 5, a little bit larger than 3.1 in Eq. 17. Third, the increasing of the force in Eq. 5 is a little slower than that in Eq.17. Fourth, the value of the first consistent at the right side in Eq. 6 is a little bit lower than the value of 0.24 in Eq. 18 . Fifth, the height of $3 h / 7$ in Japanese specification is also a little bit lower than $h / 2$ in Chinese one. One can find that the second, third, fourth and fifth differences make Chinese guidelines more conservative, by comparing the force values from the two sets of formulas. Regrettably, there is a misprint in Eq. 18, the rightmost term there must be $h b^{2}$ [1]. Fortunately, Eq.18 is seldom applied, the ratio of 3.1 and water depth $5.0 \mathrm{~m}$ require a quite large pier size, say diameter at least 15 meters, for situation with shallow water. 
One of the most significant improvements of the existing Chinese guidelines from the previous one is that the comprehensive factor has been deleted completely, since a two level design procedure is adopted. Therefore, the value of the first consistent at the right side in Eq. 16 should be multiplied by 5 . A modification of the formulas with consulting Eq. 4, Eq. 5 and Eq. 6 is suggested as following.

$$
\begin{array}{ll}
\left.\mathrm{E}_{\overline{\mathrm{W}}} 0.751 .0-\frac{\mathrm{b}}{4 h}\right) C_{i} A \xi_{h} \gamma_{w} b^{2} h / g & \text { if } \frac{b}{h} \leq 2.0 \\
\mathrm{E}_{\overline{\mathrm{W}}} 0.375 C_{i} A \xi_{h} \gamma_{w} b^{2} h / g, & \text { if } \quad 2.0<\frac{b}{h}
\end{array}
$$

where all parameters are the same as in Eq. 16. One can see that Eq. 19 is the same as Eq. 4, and Eq. 20 is conservative with a ratio value from 1.0 to 1.6 for pier size $20 \mathrm{~m}$ to $30 \mathrm{~m}$ in water depth less than 5 $\mathrm{m}$ to $7.5 \mathrm{~m}$, comparing with Eq. 5. The resultant force is not significant for a situation in this range compare with the corresponding seismic effect. These formulas are simple and convenient for highway bridge design practice.

\section{Conclusions}

The origin of formulas for seismic hydrodynamic pressure on pier in water in guidelines for seismic design of highway bridges in China is reviewed. It is clear that the formulas are simplified from an analytical solution of integrating equation on velocity potential on surface of a rigid cylinder in finite water mass in three dimensional analysis and the boundary conditions. There are two errors from a misprint and a slip up to withdraw the comprehensive factor in the process modifying the design procedures with the two level design strategy. The fact is pointed out that the formulas in Chinese guidelines are conservative in total by comparison with those in Japanese specifications. Finally, two formulas are suggested for a modification, with considering simple and convenience in design practice of highway bridges.

\section{Acknowledgements}

This work was financially supported by grant of National Key R\&D Program of China 2017YFC0806009 ; 51678540 and 51778197 of National Nature Science Foundation of China; open funds 201701 of State Key Laboratory of Bridge Engineering Structural Dynamics and Key Laboratory of Bridge Earthquake Resistance Technology, Ministry of Communications, PRC.

\section{References}

[1] Ministry of Transport of the People's Republic of China: Guidelines for Seismic Design of Highway Bridges (JTG/T B02-01-2008, in Chinese)

[2] Japan Road Association: 2012 Design Specifications for Highway Bridges, Part V Seismic Design (2012).

[3] Ministry of Transport of the People's Republic of China: Specifications of earthquake resistant design for Highway Engineering (JTJ 004-89, 1989, in Chinese).

[4] Japan Road Association: 1980 Design Specifications for Highway Bridges, Part V Seismic Design (1980).

[5] Japan Road Association: 2002 Design Specifications for Highway Bridges, Part V Seismic Design (2002). 
[6] Goto H. and K. Toki: Pro. of the 3rd World Conference on Earthquake Engineering .New Zealand, II, (1965) 